\title{
ACEITAÇÃO DA DIETA HIPOSSÓDICA COM SAL DE CLORETO DE POTÁSSIO (SAL LIGHT) EM PACIENTES INTERNADOS EM UM HOSPITAL PÚBLICO
}

\author{
ACCEPTANCE OF LOW SODIUM DIET WITH SALT POTASSIUM CHLORIDE \\ (LIGHT SALT) IN PATIENTS HOSPITALIZED IN A PUBLIC HOSPITAL
}

\author{
Kacielli Filipini ${ }^{*}$, Carolina Corrêa Gomes ${ }^{2 *}$, \\ Ana Paula Perillo Ferreira Carvalho ${ }^{3 * *}$, Liana Lima Vieira ${ }^{4^{* *}}$ \\ 1nkacielli.filipini@gmail.com, ²carolcgnutri@hotmail.com, 33anapperillo@gmail.com, ${ }^{4}$ liana_vieira@hotmail.com \\ *Universidade Federal de Goiás \\ **Hospital das Clínicas - Universidade Federal de Goiás
}

Data de entrada do artigo: 02/04/2013

Data de aceite do artigo: 02/04/2014

\section{RESUMO}

Introdução: A rejeição da dieta hipossódica pode interferir no consumo alimentar de hospitalizados. Uma das maneiras de se avaliar a aceitação dessas dietas é através da determinação do Índice de Resto-Ingestão. Objetivos: Avaliar a aceitação, em pacientes de um hospital público de Goiânia, da dieta hipossódica utilizando o sal a base de cloreto de potássio (sal light), por meio da determinaçáo do Índice de RestoIngestão e de pesquisa de satisfação. Metodologia: Estudo transversal, realizado em pacientes internados com prescriçấo de dieta hipossódica. A avaliação da aceitaçáo das dietas hipossódica padrão $(\mathrm{NaCl})$ e hipossódica com sal light foi determinada quantitativamente pelo Índice de Resto-Ingestão, durante dois dias consecutivos, avaliando-se almoço e jantar. Adotou-se como índice de aceitação satisfatório o percentual de resto-ingestão inferior a $20 \%$. Dados demográficos, clínicos e pesquisa de satisfaçáo da dieta foram verificados através de questionário. O estado nutricional foi determinado pelo Índice de Massa Corporal (IMC) e categorizado por gênero. Resultados: Entre 81 pacientes, 58\% recebiam dieta hipossódica e $42 \%$, dieta hipossódica com sal light. Por meio do IMC, observou-se excesso de peso mais prevalente no sexo feminino. O tempero da dieta foi a característica que obteve maior avaliação negativa. Conclusáo: A aceitação da dieta hipossódica com utilização do sal light foi insatisfatória, tendo a característica "tempero" da dieta avaliada negativamente pela maioria dos pacientes. Sáo necessários maiores estudos na tentativa de padronização da quantidade de sal light indicada.

Palavras-chave: dieta hipossódica; ingestão de alimentos; cloreto de potássio; serviço hospitalar de nutrição.

\section{ABSTRACT}

Introduction: The rejection of a low sodium diet can interfere with food intake of inpatients. One way to assess the acceptability of these diets is through the determination of the rest-intake index. Objectives: To assess the acceptability, in patients of a public hospital in Goiânia, of the low sodium diet using the salt-based potash (light salt), by determining the rest-intake index and satisfaction survey. Methodology: Cross-sectional study conducted in hospitalized patients with prescription of a low sodium diet. The asssessment of the acceptance of standard low sodium $(\mathrm{NaCl})$ and low sodium with light salt diets was quantitatively determined by the Rest-Intake Index, during two consecutive days, evaluating lunch and dinner. As a satisfactory acceptance rate, we adopted rest-intake percentages below 20\%. Demographic, clinical and dietary satisfaction survey of the diet were verified by questionnaire. Nutritional status was determined by Body Mass Index (BMI) and categorized by genre. Results: Among 81 patients, 58\% had a standard low sodium diet and $42 \%$, low sodium with light salt. Through the BMI, we observed overweight to be more prevalent in females. The seasoning of the diet was feature with highest negative 
rating. Conclusion: The acceptance of the low sodium diet with use of light salt was unsatisfactory, having the "seasoning" characteristic of the diet evaluated negatively by most patients. More studies are necessary in an attempt to standardize the amount of light salt indicated.

Keywords: low sodium diet; food intake; potassium chloride; hospital nutrition service.

\section{Introdução}

É crescente a preocupação com o estado nutricional do indivíduo hospitalizado, uma vez que a desnutrição intra-hospitalar representa uma causa frequente do aumento da morbimortalidade durante a internação. No Brasil, a desnutrição hospitalar atinge $48,1 \%$ dos pacientes internados na rede pública ${ }^{1}$.

A perda de peso durante a hospitalização é multifatorial, sendo consequência da ingestáo alimentar insatisfatória, resultado não só dos sintomas gastrointestinais desencadeados por doenças, mas muitas vezes do preconceito do paciente em relação à alimentação hospitalar².

A aceitação da dieta hospitalar é fundamental para suprir as necessidades nutricionais do paciente e contribuir para a recuperação e/ou manutenção de seu estado nutricional ${ }^{3}$. Determinadas enfermidades requerem implementação de um plano alimentar hipossódicón. Estudos indicam a falta de sal como uma das justificativas mais frequentes para a náo aceitaçáo das dietas que necessitam de restrição nesse componente $e^{4,5}$.

A necessidade nutricional de sódio para os seres humanos é de $500 \mathrm{mg}$ (cerca de $1,2 \mathrm{~g}$ de sal), tendo sido definida recentemente, pela Organização Mundial de Saúde (OMS), em $5 \mathrm{~g}$ de cloreto de sódio ou sal de cozinha (que corresponde a $2 \mathrm{~g}$ de sódio) a quantidade considerada máxima saudável para ingestão alimentar diária. O consumo médio do brasileiro corresponde ao dobro do recomendado ${ }^{6}$.

Apesar de necessária, essa modificação dietética não é facilmente aceita, pois o sal e a sua relação com a comida possuem representaçóes simbólicas, culturalmente estabelecidas, por tratar-se de um componente de fácil acesso, baixo custo e alto poder de condimentação ${ }^{7}$.

A rejeição da dieta hipossódica pode interferir na ingestão de energia e de macronutrientes, podendo levar a um consumo alimentar inadequado e, consequentemente, à desnutrição hospitalar ${ }^{8-10}$. Além disso, outros componentes devem ser considerados durante a abordagem nutricional, como gosto, cor, forma, aroma e textura, além da temperatura das refeiçóes, o horário de distribuição, o ambiente onde se dá a refeição, entre outros elementos ${ }^{11}$.

Para amenizar estes problemas, o nutricionista e toda a equipe de saúde devem buscar meios diferenciados para satisfazer as necessidades nutricionais de cada indivíduo, respeitando suas preferências e as limitações impostas pela doença ${ }^{12}$.
Entre as diferentes formas de reduzir o sódio da dieta, pode-se utilizar produtos comerciais com substituição do cloreto de sódio pelo cloreto de potássio, o chamado "sal light", que possui teor de sódio reduzido em até $60 \%$. A utilização desse ingrediente nem sempre é factível tanto em decorrência dos problemas de palatabilidade e intolerância gástrica dos sais de potássio quanto pela falta de hábito na ingestão alimentar deste ín $^{13}$.

Uma das maneiras de se avaliar a aceitação dessas dietas é por meio da determinação do Índice de RestoIngestão, que é a relação entre o resto de alimento devolvido pelo comensal e a quantidade de alimentos e preparaçóes alimentares oferecidas, expressa em percentual. Quando o resultado apresenta-se acima de 20\% em coletividades enfermas, pressupóe-se alguma inadequação dos cardápios, relacionados ao mau planejamento ou execuçãa ${ }^{14}$.

Diante das consideraçóes anteriores, o objetivo deste estudo foi avaliar a aceitação, em pacientes de um hospital público de Goiânia, da dieta hipossódica utilizando o sal à base de cloreto de potássio (sal light), por meio da determinação do Índice de Resto-Ingestáo e de pesquisa de satisfação, em comparação à dieta hipossódica padrão, com teor reduzido de cloreto de sódio.

\section{Metodologia}

O presente trabalho está inserido em um projeto guarda-chuva intitulado "Caracterização dos atendimentos realizados pelo Hospital das Clínicas da Universidade Federal de Goiás". Trata-se de um estudo transversal desenvolvido no Hospital das Clínicas (HC) de Goiânia, da Universidade Federal de Goiás, no período de maio a junho de 2012. O HC é um hospital universitário e conta, em sua totalidade, com 321 leitos que atendem ao Sistema Único de Saúde (SUS).

A Unidade de Produção de Refeiçôes (UPR) produz diariamente 3570 refeiçóes destinadas aos pacientes, com cardápio em padrão popular, das quais 400 são almoço e jantar. As dietas orais padronizadas na UPR recebem diferentes classificações para atender às necessidades individuais de cada paciente e são divididas segundo a consistência (livre, branda, pastosa, líquida pastosa, líquida completa e líquida restrita) e segundo sua composição (hipossódica, insuficiência renal, diabetes, hepatopata, sem irritantes gástricos etc). 
As refeiçóes da dieta hipossódica são produzidas na UPR dietética, o porcionamento do almoço e do jantar é padronizado por meio de medidas caseiras e realizado de forma centralizada. O porcionamento das refeiçóes se dá da seguinte maneira: $90 \mathrm{~g}$ de feijāo, $130 \mathrm{~g}$ de arroz, $90 \mathrm{~g}$ de prato protéico, $90 \mathrm{~g}$ de guarnição e $90 \mathrm{~g}$ de salada crua, totalizando, em média, $490 \mathrm{~g}$ por refeição. A dieta é entregue em embalagens de alumínio descartáveis tipo "marmitex".

A dieta hipossódica padrão é preparada com acréscimo de $1,5 \mathrm{~g}$ de sal de cozinha (cloreto de sódio $-\mathrm{NaCl}$ ) em cada refeição (almoço e jantar), totalizando, ao final do dia, cerca de $2 \mathrm{~g}$ de sódio.

Para o estudo, a dieta hipossódica padrão foi preparada com utilizaçáo do sal light, à base de cloreto de potássio $(\mathrm{KCl})$ LiteSalt, da marca Linea, que possui redução de $60 \%$ do teor de sódio, sendo denominada "dieta hipossódica com sal light". Para determinar a quantidade de sal light a ser utilizada, as preparaçóes do cardápio do almoço e jantar passaram por análises sensoriais, buscando alcançar sabor agradável, aliadas a uma quantidade satisfatória de sódio. Deste modo, foram acrescentados às refeiçóes $2,5 \mathrm{~g}$ de sal light, sendo adicionados $1 \mathrm{~g}$ na porção do feijáo e $0,5 \mathrm{~g}$ em cada porção de arroz, guarniçáo e carne. A quantidade total de sódio oferecida na dieta foi de $1450 \mathrm{mg}$ por dia.

O estudo foi realizado com 81 pacientes internados a mais de 24 horas, maiores de 18 anos, de ambos os sexos, com prescrição de dieta hipossódica de consistência normal ou branda. Foram determinados os pesos de 324 refeiçóes distribuídas aos pacientes nas Unidades de Internação (UI) médica, cirúrgica, ortopédica e pronto-socorro.

Foram excluídos pacientes com prescrição de dieta líquida ou pastosa, em uso de nutrição enteral ou parenteral associada, em jejum para exames ou procedimentos, com algum tipo de insuficiência renal ou restrição alimentar de potássio, amputados dos dois membros inferiores (pela dificuldade na avaliação antropométrica), impossibilitados de responder o questionário e que não estivessem acompanhados por um cuidador, internados nas Unidades de Terapia Intensiva (UTI) e/ou pertencentes a grupos vulneráveis, como crianças, gestantes e indígenas.

A avaliação da aceitação das dietas hipossódica padrão $(\mathrm{NaCl})$ e hipossódica com sal light foi determinada quantitativamente pelo Índice de Resto-Ingestão (IR), referido por Gandra e Gambardella, que estabelece a relação percentual entre o Peso da refeição rejeitada (PR) e o peso da refeição distribuída (PRD), representada pela fórmula $I R=P R \times 100 / P R D^{15}$. Adotou-se como índice de aceitação satisfatório para a população enferma o percentual de resto-ingestão inferior a $20 \%{ }^{14}$.
A coleta de dados para o cálculo do IR foi realizada por dois dias consecutivos, nas refeiçóes do almoço e do jantar das dietas hipossódicas padrão e hipossódica com sal light, a partir dos processedimentos de determinação do peso das refeiçóes distribuídas (PRD), onde foi determinado o peso, individualmente, de cada marmita já porcionada com a refeição completa, utilizando-se uma balança digital (da marca C\&F, com sensibilidade de 1 g, capacidade mínima de 25 g e máxima de $3 \mathrm{~kg}$ ), e determinação do peso dos restos das refeiçóes (PR), onde os restos obtidos das refeiçóes provenientes do almoço e jantar foram determinados da mesma maneira do procedimento inicial.

Foi aplicado, por nutricionistas treinadas, um questionário que abordou características sociodemográficas (sexo, idade, escolaridade, cor da pele), de estilo de vida (tabagismo, etilismo e prática de atividades físicas) e dados clínicos (clínica de internação, especialidade médica de origem, morbidades referidas, sintomas gastrointestinais). Foram considerados fumantes os pacientes que referiam ter o hábito de fumar ou ter parado de fumar há menos de 6 meses, e foram classificados como praticantes de atividades físicas aqueles que afirmaram praticar algum tipo de atividade física com frequência. Os sintomas gastrointestinais investigados foram náuseas, vômitos, diarreia, pirose, disfagia, odinofagia, e foi categorizada a ausência ou presença de um ou mais desses sintomas.

O questionário também abordava questões sobre percepção da dieta oferecida, onde foram avaliados indicadores como temperatura, variedade, tempero, quantidade e aparência das refeiçóes. Os participantes optaram entre bom, regular e ruim. Para a compilação dos dados, as categorias "regular' e "ruim" foram classificadas como avaliação negativa, e a categoria "bom" como avaliaçáo positiva.

Foram coletados dados antropométricos como peso, altura, circunferência braquial e dobra cutânea tricipital. Naqueles onde o peso e a altura não foram possíveis, foram coletadas a altura do joelho e a circunferência braquial, a fim de estimar altura e peso corporal através das fórmulas propostas por Chumlea, Roche e Steinbaugh ${ }^{16}$ e Chumlea et al..$^{17}$, respectivamente.

Para avaliar o estado nutricional dos pacientes foi utilizado o Índice de Massa Corporal (IMC), definido pela fórmula: peso $(\mathrm{kg}) /$ altura $(\mathrm{m})^{2}$. Para a classificação do IMC, utilizaram-se para adultos (18 a 60 anos) critérios estabelecidos pela Organização Mundial da Saúde ${ }^{18}$, e para os idosos ( $\geq 60$ anos) critérios estabelecidos por Lipschitz $^{19}$, por serem mais específicos para essa faixa etária.

Os dados obtidos foram tabulados utilizando o software Microsoft Office Excel e para análise dos dados foi utilizado o STATA/SE, versão 8.0, e o Statistical Package 
for the Social Scienes (SPSS), versão 13.0. As variáveis contínuas foram analisadas para descrição sob a forma de médias, desvios padrão e a normalidade verificada pelo teste de Kolmogorov-Smirnov. Para a comparação entre o percentual de resto-ingestão da dieta hipossódica com sal light encontrado e o percentual de aceitação satisfatório para população enferma (20\%), foi aplicado o teste t para uma amostra. Para a comparação dos valores obtidos entre as dietas avaliadas utilizou-se o teste $\mathrm{t}$ independente.

Foi calculada a frequência da aceitação para algumas variáveis analisadas. Realizou-se análise bivariada pela regressão de Poisson, apresentada pela razão de prevalência (RP) e respectivo intervalo de confiança de $95 \%$, considerando-se nível de significância de 5\%.

O projeto guarda chuva foi aprovado pelo Comitê de Ética em Pesquisas do Hospital das Clínicas da Universidade Federal de Goiás, protocolo no 078/2011. Todos os participantes assinaram o termo de consentimento livre e esclarecido antes de serem incluídos na amostra.

\section{Resultados}

Foram avaliados 81 pacientes, dos quais 58,0\% $(\mathrm{n}=47)$ recebiam dieta hipossódica padrão com $\mathrm{NaCl}$, e $42 \%(\mathrm{n}=34)$ dieta hipossódica com sal light. $\mathrm{Na}$ amostra total houve predominância do sexo masculino $(54,3 \%)$, sendo que $50,6 \%$ tinham 60 anos ou mais (Tabela 1 ).

A especialidade médica mais frequente entre os internados foi cardiologia, com $32,1 \%$. A presença de sintomas gastrointestinais durante a internação foi relatada por $53,1 \%$ dos participantes (Tabela 1 ).

Quanto aos hábitos de vida questionados, a maioria referiu não ser praticante de atividade física $(87,6 \%)$, pouco mais da metade $(55,6 \%)$ referiu consumir bebidas alcoólicas, e 63\% relataram não fumar (Tabela 1).

Em relação ao estado nutricional, observou-se uma baixa frequência de baixo peso e uma alta frequência de excesso de peso para ambos os sexos, sendo $29,5 \%$ para homens e 43,2\% para mulheres, como visto na Figura 1.

Quando comparadas às médias do IR entre a dieta hipossódica e hipossódica com sal light, observou-se que não houve diferença estatisticamente significativa entre os percentuais de IR, exceto para a refeição do jantar do primeiro dia (Tabela 2). O mesmo foi encontrado quando comparado o percentual de IR da dieta hipossódica ao sal light com o valor adotado com aceitação satisfatória para população enferma (20\%), dado esse não apresentado em tabela.

A avaliação da percepção da dieta hipossódica com sal light referente à pesquisa de satisfação - que abordou características da dieta como variedade, tempero, temperatura, quantidade e apresentação - é apresentada, em relação à dieta hipossódica com sal light, na Figura 2. Observa-se que a característica "tempero" foi avaliada de forma negativa por $58,8 \%$ dos entrevistados.

A associação entre gênero, idade, estado nutricional, presença de sintomas gastrointestinais e tabagismo não apresentou relaçẫo estatisticamente significante à aceitação da dieta hipossódica com sal light (Tabela 3).

\section{Discussão}

A dieta hipossódica com utilização de sal light apresentou melhor aceitaçáo quando comparada à dieta hipossódica padrão somente para a refeiçáo do jantar do primeiro dia. Esse achado pode ter sido influenciado pelo tipo de preparação presente no cardápio desse dia, porém esse fator não foi avaliado neste estudo.

Outro fator que pode ter interferido na melhor aceitação desta refeiçáo é a menor quantidade de alimento servida (aproximadamente $406 \mathrm{~g}$ ). Muitos pacientes queixamse de que o recipiente contém uma quantidade excessiva de alimento e que isto interfere negativamente na aceitação.

Vieira et al. ${ }^{20}$, em estudo da aceitabilidade de saladas pela adição de óleos aromatizados em UPR hospitalar, encontraram que um dos fatores que podem modificar o consumo alimentar é a oferta de porçóes muito grandes. Por isso, é necessário conhecer o hábito alimentar da população atendida para diminuir a quantidade de alimento rejeitada. Um Índice de Resto-Ingestão elevado pode ser decorrência de falha no porcionamento das refeiçôes, apresentação desfavorável e preparaçôes incompatíveis com hábitos ou padrōes alimentares dos paciente ${ }^{21,22}$.

Neste estudo, a pesquisa de satisfação obteve avaliação negativa para a característica "tempero" para a maioria dos pacientes, sendo a apresentação, temperatura, variedade e quantidade da dieta avaliados positivamente. Apesar de este estudo ter obtido avaliaçóes positivas para temperatura, essa característica foi associada à baixa aceitação na pesquisa de Stanga et al..$^{23}$, onde mais de $70 \%$ dos pacientes consideraram a temperatura como aspecto mais relevante na aceitação da dieta.

Yabuta et al. avaliaram a aceitação da dieta hipossódica em pacientes hospitalizados e encontraram como fatores para aceitação negativa a falta de sal $(21,1 \%)$ e a insatisfação quanto ao sabor da refeição $(11,5 \%)$. Estudos realizados com pacientes hospitalizados obtiveram as menores notas atribuídas às refeiçóes de almoço e jantar para o atributo sabor/tempero ${ }^{3,24}$. Um estudo que avaliou a aceitação de dieta hipossódica hospitalar observou que mesmo entre pacientes que relataram aceitar plenamente a refeição oferecida, $82 \%$ sentiram falta do sal na refeição, e $52 \%$, a falta de temperos ${ }^{4}$. 
Tabela 1: Características sociodemográficas, antropométricas, dados clínicos e de estilo de vida dos pacientes internados no Hospital das Clínicas ( $\mathrm{n}=81$ )

\begin{tabular}{|c|c|c|c|}
\hline Variável & $\begin{array}{l}\text { Total } \\
\text { n }(\%)\end{array}$ & $\begin{array}{c}\text { Hipossódica Sal Light }(\mathbf{n}=34) \\
\text { n }(\%)\end{array}$ & $\begin{array}{c}\text { Hipossódica } \\
\text { Padráo }(n=47) \\
\text { n }(\%)\end{array}$ \\
\hline \multicolumn{4}{|l|}{ Sexo } \\
\hline Feminino & $37(45,68)$ & $15(44,12)$ & $22(46,81)$ \\
\hline Masculino & $44(54,32)$ & $19(55,88)$ & $25(53,19)$ \\
\hline \multicolumn{4}{|l|}{ Idade (anos) } \\
\hline $18-59$ & $40(49,38)$ & $17(50,00)$ & $23(38,94)$ \\
\hline$\geq 60$ & $41(50,62)$ & $17(50,00)$ & $24(61,06)$ \\
\hline \multicolumn{4}{|l|}{ Estado nutricional } \\
\hline Baixo peso & $12(14,81)$ & $8(23,53)$ & $4(8,51)$ \\
\hline Eutrofia & $40(49,38)$ & $11(32,35)$ & $29(61,70)$ \\
\hline Excesso de peso & $29(35,81)$ & $15(44,12)$ & $14(29,79)$ \\
\hline \multicolumn{4}{|l|}{ Especialidade médica } \\
\hline Cardiologia & $26(32,10)$ & $12(35,29)$ & $14(29,79)$ \\
\hline Cirurgia vascular & $8(9,89)$ & $3(8,82)$ & $5(10,64)$ \\
\hline Cirurgia geral & $7(8,64)$ & $3(8,82)$ & $4(8,51)$ \\
\hline Urgência adulto & $3(3,65)$ & $3(8,82)$ & - \\
\hline Pneumologia & $11(13,60)$ & $3(8,82)$ & $8(17,01)$ \\
\hline Gastroenterologia & $7(8,64)$ & $2(5,88)$ & $5(10,64)$ \\
\hline Medicina interna & $2(2,47)$ & $2(5,88)$ & - \\
\hline Nefrologia & $4(4,94)$ & - & $4(8,51)$ \\
\hline Endocrinologia & $2(2,47)$ & - & $2(4,26)$ \\
\hline Outras & $11(13,60)$ & $6(17,65)$ & $5(10,64)$ \\
\hline \multicolumn{4}{|c|}{ Sintomas gastrointestinais } \\
\hline Presença & $43(53,09)$ & $14(41,18)$ & $29(61,70)$ \\
\hline Ausência & $38(46,91)$ & $20(58,82)$ & $18(38,30)$ \\
\hline \multicolumn{4}{|c|}{ Prática de atividade física } \\
\hline Não & $71(87,65)$ & $31(91,18)$ & $40(85,11)$ \\
\hline Sim & $10(12,35)$ & $3(8,82)$ & $7(14,89)$ \\
\hline \multicolumn{4}{|l|}{ Tabagismo } \\
\hline Fumante/ex-fumante & $30(37,04)$ & $13(38,24)$ & $17(36,17)$ \\
\hline Não fumante & $51(62,96)$ & $21(61,76)$ & $30(63,83)$ \\
\hline \multicolumn{4}{|l|}{ Bebida alcoólica } \\
\hline Consomem & $45(55,55)$ & $6(17,65)$ & $8(17,02)$ \\
\hline Não consomem & $36(44,45)$ & $28(82,35)$ & $39(82,98)$ \\
\hline
\end{tabular}

A avaliação negativa quanto ao quesito tempero pode estar relacionada à utilização do sal à base de $\mathrm{KCl}$ (sal light), uma vez que a ingestão alimentar deste íon ainda não é um hábito alimentar da nossa população. $\mathrm{O}$ uso do sal light ainda náo é bem descrito na literatura, e existem relatos de problemas relacionados à palatabilidade dos sais de potássio ${ }^{13}$.

Quanto à avaliaçáo do estado nutricional, observou-se uma alta prevalência de excesso de peso para ambos os gêneros, sendo $29,5 \%$ para homens e $43,2 \%$ para mulheres, superando a prevalência de baixo peso comumente encontrada em pacientes hospitalizados. Uma prevalência ainda maior foi encontrada em um estudo que avaliou o estado nutricional de pacientes em dieta hipossódica com tratamento ambulatorial, onde $66,6 \%$ e $63,9 \%$ dos homens e mulheres, respectivamente, apresentavam algum grau de excesso de peso ${ }^{25}$. A prevalência de excesso de peso pode estar relacionada ao alto índice de sedentarismo encontrado nesse estudo, bem como ao próprio perfil dos pacientes, que muitas vezes possuem doenças crônicas diretamente relacionadas ao sobrepeso e à obesidade.

Quanto aos hábitos de vida, foram encontrados altos níveis de sedentarismo $(87,6 \%)$, consumo de bebidas alcoólicas $(55,6 \%)$ e tabagismo $(37,0 \%)$. Figueiredo e Asakura encontraram uma taxa de sedentarismo de $48,2 \%$ e uma baixa prevalência de tabagismo $(5,6 \%)$ e consumo de 
Tabela 2: Comparação da média do percentual de resto-ingestão da dieta hipossódica com sal light e dieta hipossódica padrão

\begin{tabular}{|c|c|c|c|}
\hline & Média (\%) & Desvio Padráo (\%) & Valor $\mathrm{p}^{*}$ \\
\hline Almoço do primeiro dia & & & 0,059 \\
\hline Hipossódica Sal Light & 34,687 & 27,738 & \\
\hline Hipossódica padrão & 46,861 & 28,763 & \\
\hline Jantar do primeiro dia & & & 0,04 \\
\hline Hipossódica Sal Light & 29,186 & 25,396 & \\
\hline Hipossódica padrão & 46,699 & 27,935 & \\
\hline Almoço do segundo dia & & & 0,526 \\
\hline Hipossódica Sal Light & 40,787 & 28,781 & \\
\hline Hipossódica padrão & 44,913 & 28,706 & \\
\hline Jantar do segundo dia & & & 0,124 \\
\hline Hipossódica Sal Light & 38,325 & 30,000 & \\
\hline Hipossódica padrão & 48,775 & 29,658 & \\
\hline
\end{tabular}

*Teste $\mathrm{t}$ independente.

Tabela 3: Frequência de aceitação insatisfatória e fatores associados segundo características sociodemográficas, estilo de vida e condições de saúde de pacientes hospitalizados recebendo dieta hipossódica com sal light $(\mathrm{N}=34)$

\begin{tabular}{lccc}
\hline Variáveis & $\begin{array}{c}\text { Frequência } \\
\mathbf{n}(\%)\end{array}$ & RP (IC95\%) & Valor $\mathbf{p}^{*}$ \\
\hline Sexo & $14(93,33)$ & $1,26(0,76-2,12)$ & 0,368 \\
Feminino & $10(52,63)$ & 1,0 & \\
$\begin{array}{l}\text { Masculino } \\
\text { Faixa Etária }\end{array}$ & $13(76,47)$ & $1,07(0,64-1,79)$ & 0,793 \\
Adultos & $11(64,71)$ & 1,0 & \\
Idosos & & $1,09(0,57-2,12)$ & 0.790 \\
Estado nutricional & $6(75,00)$ & $1,14(0,63-2,06)$ & 0.673 \\
Baixo peso & $9(81,82)$ & 1,0 & \\
Eutrofia & $9(60,00)$ & 1,0 & \\
Excesso de peso & & $1,23(0,72-2,11)$ & \\
Sintomas gastrointestinais & $7(50,00)$ & $1,22(0,73-2,06)$ & 0,443 \\
Nenhum & $17(85,00)$ & 1,0 & \\
1 ou mais & & \\
Tabagismo & $12(92,31)$ & & \\
Fumante & $12(54,14)$ & & \\
Náo fumante & & & \\
\hline
\end{tabular}

*Teste $\mathrm{t}$ independente.

bebidas alcoólicas (16,7\%). Não foi encontrada relação entre a aceitação da dieta com os hábitos de vida dos pacientes, mas estudos recentes demonstram que o cigarro causa prejuízos, mesmo que de forma imperceptível, no reconhecimento olfativo e gustativo de fumantes ${ }^{26}$.

$\mathrm{O}$ presente estudo não encontrou associação entre sexo, idade, estado nutricional, tabagismo e presença de sintomas gastrointestinais e a aceitação insatisfatória da dieta hipossódica com uso de sal light. Molero et al. avaliaram a aceitação de dietas hospitalares consideradas "normais" e também não encontraram associação entre sexo e aceitaçáo de dieta ${ }^{27}$. Outro estudo observou que os homens apresentaram uma melhor aceitação da dieta hipossódica, sugerindo que as mulheres têm mais dificuldade em aceitar a alimentação com restrição de sal do que os homens ${ }^{4}$. Outro estudo demonstrou que existe influência do gênero e idade sob a aceitação de dietas hospitalares ${ }^{3}$.

Zazzo e Puissant observaram que os problemas relacionados ao consumo de dietas hospitalares estavam ligados à doença ou ao tratamento em 32,1\% dos casos ${ }^{28}$. 
Figura 1: Classificação do estado nutricional pelo IMC segundo gênero dos pacientes recebendo dieta hipossódica padrão e hipossódica com sal light internados em um hospital público $(\mathrm{n}=81)$

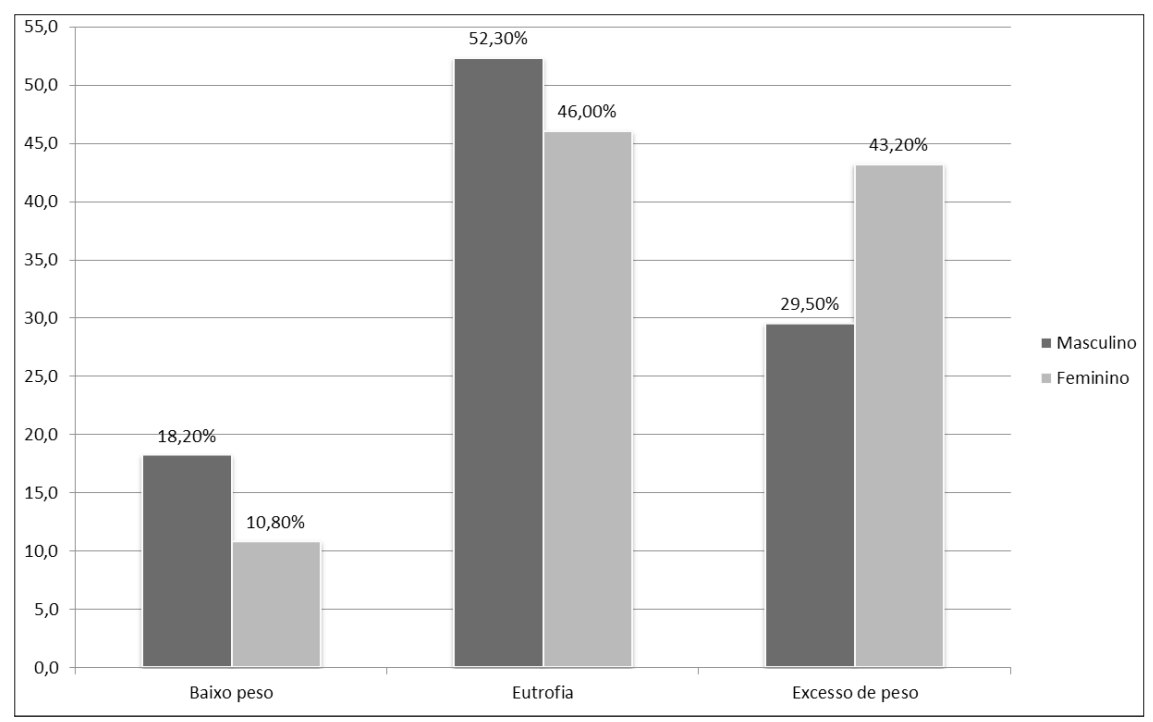

Figura 2: Avaliação da percepção da dieta hipossódica com sal light por pacientes internados no Hospital das Clínicas $(n=34)$

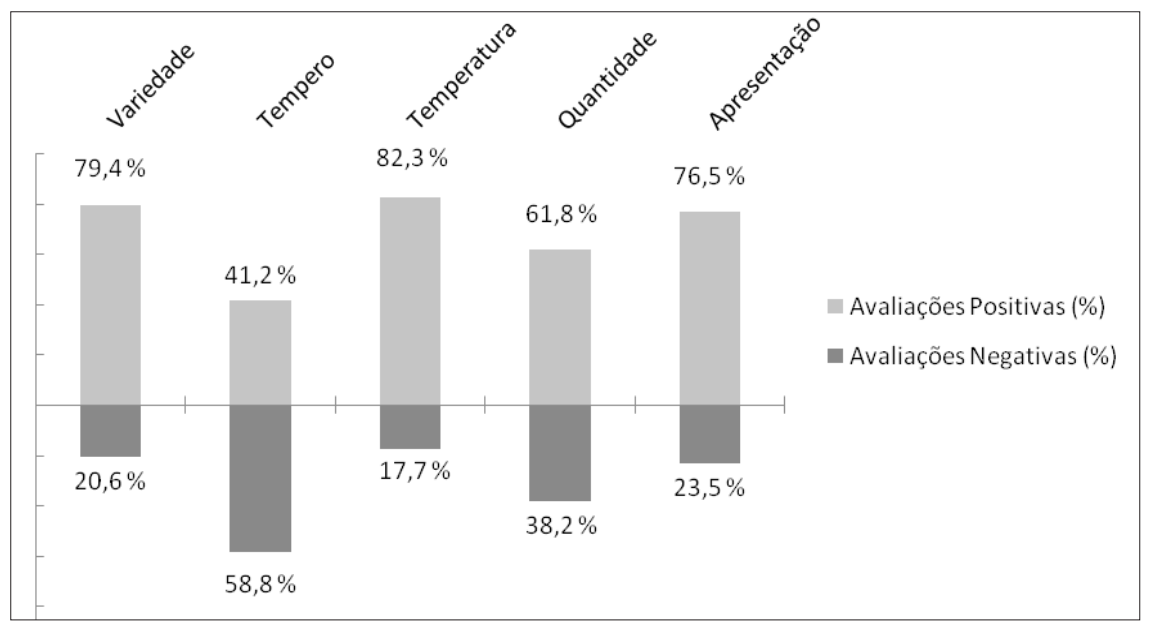

Assim, mesmo que o presente estudo não tenha observado associação entre a presença de sintomas gastrointestinais e a aceitação insatisfatória, este fator pode ser um agravante para a baixa aceitação da dieta durante $o$ período de internação.

Entre as limitaçóes do estudo, pode-se citar o tempo de coleta do resto-ingestão, levando-se em conta que um maior tempo de coleta pode mostrar as alteraçóes referentes ao maior tempo de internação, dado que existe uma relação negativa entre tempo de internaçáo e aceitação de dieta. $\mathrm{O}$ tipo de estudo náo permitiu um acompanhamento do paciente em relação à aceitação da dieta e do estado nutricional. $\mathrm{O}$ tamanho reduzido da amostra também pode ter interferido nos resultados, bem como a falta de detalhamento das preparaçóes utilizadas nas dietas durante o tempo de coleta de dados.

No ambiente hospitalar, a baixa aceitação da dieta hipossódica torna-se mais relevante. Devem-se buscar formas de amenizar a falta de sabor dessas dietas, priorizando a personalização e a melhora da aparência, aroma, textura e sabor ${ }^{29}$. São diversos os fatores que influenciam essa aceitação, desta forma, é imprescindível que haja preocupaçấo e controle em todas as fases da produçáo de alimentos, do planejamento ao preparo e à distribuição. 


\section{Conclusão}

A aceitação da dieta hipossódica com utilização do sal light foi insatisfatória, tendo a característica "tempero" da dieta avaliada negativamente pela maioria dos pacientes. São necessários maiores estudos na tentativa de padronização da quantidade de sal à base de $\mathrm{KCl}$ (sal light) indicada, buscando um equilíbrio entre quantidade de sódio e potássio e o sabor das preparaçóes para que possamos utilizá-lo como substituto do cloreto de sódio e assim melhorar a aceitação das dietas restritas nesse elemento. Deve-se ampliar a visão sobre dietas hospitalares, buscar aliar a prescrição dietética e as restrições alimentares e proporcionar refeiçôes saborosas e atrativas. Sendo assim, cabe ao nutricionista utilizar técnicas dietéticas adequadas para aumentar a adesão dos pacientes.

\section{Referências}

1. Waitzberg DL, Caiaffa WT, Correia ITD. Hospital malnutrition: the Brazilian national survey (IBRANUTRI): a study of 4000 patients. Nutrition. 2001;17(7-8):573-80.

2. Prieto DB, Leandro-Merhi VA, Mônaco DV, Lazarini ALG. Intervenção nutricional de rotina em pacientes de um hospital privado. Rev Bras Nutr Clin. 2006;21(3):181-7.

3. Coloço RB, Holanda LB, Portero-Mclellan KC. Determinantes do grau de satisfação de pacientes internados referente a refeiçóes oferecidas em um hospital universitário. Rev Ciênc Méd. maio/jun. 2009;18(3):121-30.

4. Santos BF, Cammerer MA, Marcadenti A. Aceitação de dietas com reduzido teor de sódio entre cardiopatas em um hospital terciário. Rev Ciência \& Saúde. jul./dez. 2012;5(2):79-86.

5. Sociedade Brasileira de Cardiologia; Sociedade Brasileira de Hipertensão; Sociedade Brasileira de Nefrologia. VI Diretrizes Brasileiras de Hipertensão. Arq Bras Card. 2010;95(1):1-51.

6. Dallepiane LB, Bós AJG. O uso de condimentos na dieta em um grupo de hipertensos: estudo de intervenção randomizado. Departamento de Ciências da Saúde da Unijuí. 2007.

7. Thomas RD, Zdrowski CD, Wilson MM, Conright KC, Lewis C, Tariq S, et al. Malnutrition in subacute care. Am J Clin Nutr. 2002;75:308-13.

8. Bentley B, Jong JM, Moser KD, Peden RA. Factors related to noadherence to low sodium diet recommendations in heart failure patients. Eur J Cardiovasc Nurser. 2005;4:331-6.

9. Sousa AA, Gloria MS, Cardoso TS. Aceitação de dietas em ambiente hospitalar. Rev Nutr. 2011;24(2):287-94.

10. Parisenti J, Copetti Firmino C, Espíndola Gomes C. Avaliação de sobras de alimentos em unidade produtora de refeiçóes hospitalares e efeitos da implantação do sistema de hotelaria. Alim Nutr. abr./jun. 2008;19(2):191-4.
11. Lotaif LAD, Kohlmann Junior O, Zanella MT, Kohlmann NEB, Ribeiro AB. Efeito da suplementação de potássio através do sal de cozinha na hipertensão arterial primaria leve a moderada. J Bras Nefrol. 1995;17(4):214-8.

12. Castro MDAS, Oliveira LF, Silva LPRB. Resto-Ingesta e aceitação de refeiçóes em uma Unidade de Alimentaçáo e Nutrição. Hig Aliment. 2003;17(114-115):24-8.

13. Gandra YR, Gambardella AMD. Avaliação de serviços de Nutrição e Alimentação. São Paulo: Sarvier; 1986.

14. Chumlea WC, Roche AF, Steinbaugh ML. Estimating stature from knee height for persons 60 to 90 years of age. J Am Geriatr Soc. 1985;33(2):116-20.

15. Chumlea WC, Roche AF, Steinbaugh ML. Prediction of body weight for the nonambulatory elderly from athropometry. J Am Diet Assoc. 1988;88(5):564-8.

16. World Health Organization. Obesity. Preventing and managing the global epidemic. Report of a WHO consultation on obesity. WHO/NUT/NCD/981. Geneva: WHO; 1998.

17. Lipschitz DA. Screening for nutritional status in the elderly. Primary care. 1994;21(1):55-67.

18. Vieira FGK, Balestrin ELB, Sousa AA, Tasca CG. Implementação de óleos aromatizados em saladas: avaliação de aceitabilidade em uma unidade produtora de refeiçóes hospitalares. Hig Alim. 2007;21(149):30-4.

19. Almdal T, Viggers L, Beck AM, Jensen K. Food production and wastage in relation to nutritional intake in a general district hospital: wastage is not reduced by training the staff. Clin Nutr. 2003;22(1):47-51.

20. Barton AD, Beigg CL, MacDonald IAP, Allison S. High food wastage and low nutritional intakes in hospital patients. Clin Nutr. 2000;19(6):445-9.

21. Stanga Z, Zurfluh Y, Roselli M, Sterchi AB, Tanner B, Knecht G. Hospital food: a survey of patients perceptions. Clin Nutr. 2003; 23(3):241-6.

22. Santos GRB. Aceitação da dieta hipossódica por pacientes hipertensos internados em um hospital no município de São Bernardo do Campo - SP. Rev Bras Ciên Saúde. 2007;13(ano III):33-40.

23. Figueiredo NN, Asakura L. Adesão ao tratamento anti-hipertensivo: dificuldades relatadas por indivíduos hipertensos. Acta Paul Enferm. 2010;23(6):782-7.

24. Santos KW, Vidor DCGM, Maahs MAP. Preliminary studies comparing smokers and nonsmokers about the smell and taste perception. Int Arch Otorhinolaryngol. 2012;16(Suppl. 1):106.

25. Molero G, Fuster GO, Liébana MI, Oliva L, López ML, Aguilar AM. Influencia de la temperatura en la ingesta de pacientes hospitalizados. Nutr Hosp. 2008;23(1):54-9.

26. Zazzo JF, Puissant MC, Aubert P. Nutritional status and food intake in adult, pediatric and elderly hospitals: a 4-days survey in Assistance Publique-Hôpitaux de Paris. Nutr Clin Metabol . 2003;17(4):213-7.

27. Ginani V. Araújo W. Gastronomia e dietas hospitalares. Nutr Pauta. 2002;53:17-21. 